\title{
Exercise-based cardiac rehabilitation in twelve European countries results of the European cardiac rehabilitation registry is
}

\author{
${ }^{a}$ Reha Sports Institute and Case Management Centre, Feldkirch, Austria \\ b Institut für Herzinfarktforschung, Ludwigshafen, Germany \\ c Department of Cardiology, Spital Tiefenau, Bern, Switzerland \\ d National Center of Rehabilitation and Palliation, University of Southern Denmark and University Hospital Odense, Denmark \\ e Hasselt University and Hartcentrum Hasselt, Belgium \\ ${ }^{\mathrm{f}}$ CV Research Laboratory, Biomedical Research Foundation, Academy of Athens, Athens, Greece \\ g Sports Medicine Laboratory, Aristotle University of Thessaloniki, Thessaloniki, Greece \\ h State Hospital for Cardiology, Balatonfured, Hungary

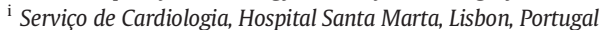 \\ ${ }^{\mathrm{j}}$ Federal Health Center and National Center for Preventive Medicine, Moscow, Russia \\ ${ }^{k}$ Cardiac Rehabilitation Clinic, University of Medicine and Pharmacy, Timisoara, Romania. \\ ${ }^{1}$ Clinic for Diagnostic, Rehabilitation and Prevention of CV Diseases, Thalassotherapia Opatija, Croatia \\ m Park Klinikum Lazariterhof, Bad Krozingen, Germany \\ n Department of Psychology, Royal College of Surgeons in Ireland, Dublin, Ireland
}

Werner Benzer ${ }^{\mathrm{a}, *}$, Bernhard Rauch ${ }^{\mathrm{b}}$, Jean-Paul Schmid ${ }^{\mathrm{c}}$, Ann Dorthe Zwisler ${ }^{\mathrm{d}}$, Paul Dendale ${ }^{\mathrm{e}}$, Constantinos H. Davos ${ }^{\mathrm{f}}$, Evangelia Koudi ${ }^{\mathrm{g}}$, Attila Simon ${ }^{\mathrm{h}}$, Ana Abreu ${ }^{\mathrm{i}}$, Nana Pogosova ${ }^{\mathrm{j}}$, Dan Gaita ${ }^{\mathrm{k}}$, Bojan Miletic ${ }^{1}$, Gerd Bönner ${ }^{\mathrm{m}}$, Taoufik Ouarrak ${ }^{\mathrm{b}}$, Hannah McGee $^{\mathrm{n}}$, on behalf of the EuroCaReD study group:

\section{A R T I C L E I N F O}

\section{Article history:}

Received 2 May 2016

Received in revised form 28 September 2016

Accepted 5 November 2016

Available online 11 November 2016

\section{Keywords:}

Cardiac rehabilitation

Patient selection

Cardiovascular prevention programmes

Internet-based survey for cardiovascular disease

Guideline adherence

Quality assurance

Bench marking

\begin{abstract}
A B S T R A C T
Aim: Results from EuroCaReD study should serve as a benchmark to improve guideline adherence and treatment quality of cardiac rehabilitation (CR) in Europe.

Methods and results: Data from 2.054 CR patients in 12 European countries were derived from 69 centres. $76 \%$ were male. Indication for CR differed between countries being predominantly ACS in Switzerland (79\%), Portugal (62\%) and Germany (61\%), elective PCI in Greece (37\%), Austria (36\%) and Spain (32\%), and CABG in Croatia and Russia (36\%). A minority of patients presented with chronic heart failure (4\%). At CR start, most patients already were under medication according to current guidelines for the treatment of CV risk factors. A wide range of CR programme designs was found (duration 3 to 24 weeks; total number of sessions 30 to 196). Patient programme adherence after admission was high (85\%). With reservations that eCRF follow-up data exchange remained incomplete, patient $\mathrm{CV}$ risk profiles experienced only small improvements. $\mathrm{CR}$ success as defined by an increase of exercise capacity $>25 \mathrm{~W}$ was significantly higher in young patients and those who were employed. Results differed by countries. After CR only $9 \%$ of patients were admitted to a structured post-CR programme. Conclusions: Clinical characteristics of $\mathrm{CR}$ patients, indications and programmes in Europe are different. Guideline adherence is poor. Thus, patient selection and CR programme designs should become more evidence-based. Routine eCRF documentation of $\mathrm{CR}$ results throughout European countries was not sufficient in its first application because of incomplete data exchange. Therefore better adherence of CR centres to minimal routine clinical standards is requested.
\end{abstract}

(c) 2016 Elsevier Ireland Ltd. All rights reserved.

\footnotetext{
is Plans for and progress on this project were presented previously at several abstract sessions of the EuroPRevent Congress 2011, 2012 and 2013

* Corresponding author at: FESC, Reha Sports Institute - Case Management Centre, Grenzweg 10, 6800 Feldkirch, Austria.

E-mail address: wbenzer@cable.vol.at (W. Benzer).
}

\section{Introduction}

Cardiovascular (CV) disease is the leading cause of death. It is responsible for almost a quarter of the disease burden in Europe resulting in substantial direct and indirect healthcare costs [1]. However, the enormous engagement in the development and availability of high technology diagnostic and therapeutic procedures for treatment of CV disease in recent decades is associated with increased survival and 
reduced age-adjusted mortality in many European countries [2]. Since CV conditions are chronic and often reflect long-term patterns of unhealthy lifestyles and/or deconditioning of patients, benefit is not automatically achieved through high technology interventions and pharmacological management alone. Several studies suggest that preventive measures are responsible for at least half of the improvement [3]. Therefore patients need to be professionally supported to regain or maintain physical capacity and to achieve changes in lifestyle, risk factor management, better well-being and social and vocational participation [4]. Cardiac rehabilitation (CR) is the structured and multicomponent intervention to deliver these services [5].

Evidence of the benefits of CR has been well established [6-8]. In its consequence $\mathrm{CR}$ is categorised as a Class I recommendation in the ACRF/ AHA guidelines for the management of patients with ST-elevation myocardial infarction [9], and a Class IIa recommendation in the ESC Guidelines for patients after acute myocardial infarction [10].

Effectiveness of $\mathrm{CR}$, however, strongly depends on minimal standards to be delivered and guaranteed during all day care in clinical practice. Therefore the need for a continuous and interactive quality assurance process is crucial. An effective quality assurance, however, only can be achieved by a regular and structured exchange of institutional and clinical data including their continuous scientific evaluation. The first representative evaluation of CR activities in European Union Member States was the Carinex Survey published in 2002 [11]. In 2008 the EACPR introduced the European Cardiac Rehabilitation Inventory Survey (ECRIS) to investigate the status of CR in European countries [12]. The ECRIS study provided information on the CR structure, legislation, funding mechanisms and national guidelines. However, neither Carinex nor ECRIS was designed to deliver information about baseline clinical variables and outcome data of patients admitted to CR. But the success of CR service provision depends on data collection and quality assessment provided by a common, international core database and data standard across Europe.

Therefore the European Cardiac Rehabilitation Registry and Database (EuroCaReD) was introduced as the next step after Carinex and ECRIS to get information about CR across Europe from a predominantly clinical perspective. Cardiology Audit and Registration Data Standards (CARDS) for Europe and collection of a common core dataset across CR centres in European countries has been promoted earlier [13]. Based on this core dataset EuroCaReD aimed to assess the current $\mathrm{CR}$ practice in clinical all day care using a web-based data collection system.

The purpose of the EuroCaReD project was to put together information on the clinical status of CR across European countries by using an electronic case report form (eCRF) to consider how this data match in different countries and what parts of the CR have to be better standardised in accordance with the current guidelines to improve treatment results.

\section{Methods}

\subsection{Study aims and characteristics}

The aims were to develop and test the feasibility and practicability of a web-based registry in European countries that routinely provides data on CR settings, contents and interventions, clinical characteristics of patients and outcomes and thereby serves as basis for a regular European quality assessment of $\mathrm{CR}$ services and results. Participation to EuroCaReD was voluntary.

EuroCaReD was designed as a central internet database (http:// www.eurocared.org) being connected to national registries and local databases of individual CR centres. The database itself was located in the European Heart House, Sophia Antipolis, France, hosted by Solarys IT Company, Götzis, Austria. All data were collected electronically using a web-based data entry system. The datasets were based on the CARDS model [13], and include items reflecting characteristics of the individual institution, patient's characteristics and actual CR performance standards [14].

\subsection{Enrolment of study population}

All European countries being members of the European Association for Cardiovascular Prevention and Rehabilitation (EACPR) have been invited to be part of the survey and 69 CR centres in 12 European countries could be selected by the national study coordinators to participate. Following the "snapshot" design of this study, for consecutive enrolment of patients undergoing CR a predefined time window of 8 weeks has initially been arranged. Because of insufficient patient inclusion within the first time period, a second prolonged time period of another 24 weeks has been offered to the participating CR centres for data collection. Except of Germany, participating only the second period, all centres collected their data within both time frames (October 1st, 2010 to November 30th, 2010, and October 1st, 2011 to February 28th, 2012). Informed consent of all participating patients was obtained according to the national regulations of the participating country.

\subsection{Electronic case report form (eCRF)}

The selection of variables aimed to closely mirror CR all day care and included the initiating clinical event (Table 1), demographic details (age, gender, employment status), history of CV risk factors (hypertension, hyperlipidaemia, diabetes, overweight/obesity, smoking, physical inactivity, depression) (Table 2), and CV risk factors as evaluated at the CR start and CR end (blood pressure, LDL-cholesterol, fasting glucose, body mass index, smoking status, watts achieved during exercise testing) (Table 4). Current medication at CR start has also been evaluated (Table 3). All these items have been tested for conformity in the participating countries. A complete overview on all eCRF items is given in Appendix 1.

\subsection{Follow-up}

Follow-up was limited to the duration of the individual rehabilitation programme, which varied considerably between 3 weeks (Hungary, Germany) and 24 weeks (Greece). Clinical follow-up data entered into the EuroCaReD database at the end of the regular CR programme included clinical events during $\mathrm{CR}$, premature ending of the CR-programme, current risk factors, exercise capacity and medication.

\subsection{Testing CR success}

Due to limited follow-up time and data acquisition not monitored by an independent clinical research organisation (CRO), CR success could not be evaluated by its effect on clinical prognosis. Moreover, as management of CV risk factors like hypertension, hyperlipidaemia and diabetes already is started by therapeutic attempts preceding CR these items cannot serve as prognostic surrogate parameters. From this background and because all participating centres were offering bicycle exercise training as a major programme content, CR success has retrospectively been defined as "exercise capacity" gained during the $C R$ process. Therefore, $C R$ was regarded to be successful, if the "gain of exercise capacity" during CR was $>25 \mathrm{~W}$ from CR start. This assumption is based on the experience in clinical practice and the need to reflect a heterogeneous population with a large variety of exercise capacities at baseline.

\subsection{Data management}

Data were anonymously entered online into the eCRF at each individual study site and stored in the central EuroCaReD database. To maintain patient's anonymity, only the identification number of each study participant was transferred to the central database. Patient's re-identification 
Table 1

Clinical events and diagnoses leading to CR referral.

\begin{tabular}{|c|c|c|c|c|c|c|c|c|c|}
\hline Country & $\begin{array}{l}\text { Total } \\
\text { number of } \\
\text { patients } \\
\text { recruited }\end{array}$ & $\begin{array}{l}\text { UAP } \\
(\%)^{\mathrm{a}} \\
\text { (numbers) }^{\mathrm{b}}\end{array}$ & $\begin{array}{l}\text { NSTEMI } \\
(\%)^{\mathrm{a}} \\
\text { (numbers) }^{\mathrm{b}}\end{array}$ & $\begin{array}{l}\text { STEMI } \\
(\%)^{\mathrm{a}} \\
\text { (numbers) }^{\mathrm{b}}\end{array}$ & $\begin{array}{l}\text { Elective PCI } \\
(\%)^{\mathrm{a}} \\
\text { (numbers) }^{\mathrm{b}}\end{array}$ & $\begin{array}{l}\text { CABG } \\
(\%)^{\mathrm{a}} \\
\text { (numbers) }^{\mathrm{b}}\end{array}$ & $\begin{array}{l}\text { Non CABG } \\
\text { cardiac } \\
\text { surgery } \\
(\%)^{\mathrm{a}} \\
\text { (numbers) }^{\mathrm{b}}\end{array}$ & $\begin{array}{l}\text { Chronic } \\
\text { heart failure } \\
(\%)^{\mathrm{a}} \\
\text { (numbers) }^{\mathrm{b}}\end{array}$ & $\begin{array}{l}\text { Othersc } \\
(\%)^{\mathrm{a}} \\
\text { (numbers) }^{\mathrm{b}}\end{array}$ \\
\hline Austria & 544 & 0.0 & $\begin{array}{l}17.4 \\
(51 / 293)\end{array}$ & $\begin{array}{l}21.8 \\
(64 / 293)\end{array}$ & $\begin{array}{l}36.5 \\
(107 / 293)\end{array}$ & $\begin{array}{l}8.9 \\
(26 / 293)\end{array}$ & $\begin{array}{l}7.5 \\
(22 / 293)\end{array}$ & $\begin{array}{l}5.5 \\
(16 / 293)\end{array}$ & $\begin{array}{l}2.4 \\
(7 / 293)\end{array}$ \\
\hline Belgium & 232 & $\begin{array}{l}0.4 \\
(1 / 225)\end{array}$ & $\begin{array}{l}10.2 \\
(23 / 225)\end{array}$ & $\begin{array}{l}16.9 \\
(38 / 225)\end{array}$ & $\begin{array}{l}24.9 \\
(56 / 225)\end{array}$ & $\begin{array}{l}20.0 \\
(45 / 225)\end{array}$ & $\begin{array}{l}14.7 \\
(33 / 225)\end{array}$ & $\begin{array}{l}10.2 \\
(23 / 225)\end{array}$ & $\begin{array}{l}2.7 \\
(6 / 225)\end{array}$ \\
\hline Croatia & 93 & $\begin{array}{l}0.0 \\
(0 / 92)\end{array}$ & $\begin{array}{l}10.9 \\
(10 / 92)\end{array}$ & $\begin{array}{l}29.3 \\
(27 / 92)\end{array}$ & $\begin{array}{l}3.3 \\
(3 / 92)\end{array}$ & $\begin{array}{l}35.9 \\
33 / 92\end{array}$ & $\begin{array}{l}19.6 \\
(18 / 92)\end{array}$ & $\begin{array}{l}0.0 \\
(0 / 92)\end{array}$ & $\begin{array}{l}1.1 \\
(1 / 92)\end{array}$ \\
\hline Denmark & 153 & $\begin{array}{l}4.1 \\
(6 / 148)\end{array}$ & $\begin{array}{l}18.9 \\
(28 / 148)\end{array}$ & $\begin{array}{l}18.2 \\
(27 / 148)\end{array}$ & $\begin{array}{l}29.7 \\
(44 / 148)\end{array}$ & $\begin{array}{l}17.6 \\
(26 / 148)\end{array}$ & $\begin{array}{l}2.7 \\
(4 / 148)\end{array}$ & $\begin{array}{l}2.7 \\
(4 / 148)\end{array}$ & $\begin{array}{l}6.1 \\
(9 / 148)\end{array}$ \\
\hline Germany & 204 & $\begin{array}{l}3.0 \\
(6 / 199)\end{array}$ & $\begin{array}{l}22.1 \\
(44 / 199)\end{array}$ & $\begin{array}{l}39.2 \\
(78 / 199)\end{array}$ & $\begin{array}{l}1.0 \\
(2 / 199)\end{array}$ & $\begin{array}{l}20.1 \\
(40 / 199)\end{array}$ & $\begin{array}{l}8.5 \\
(17 / 199)\end{array}$ & $\begin{array}{l}2.0 \\
(4 / 199)\end{array}$ & $\begin{array}{l}4.5 \\
(7 / 199)\end{array}$ \\
\hline Greece & 139 & $\begin{array}{l}0.0 \\
(0 / 139)\end{array}$ & $\begin{array}{l}0.0 \\
(0 / 139)\end{array}$ & $\begin{array}{l}3.6 \\
(5 / 139)\end{array}$ & $\begin{array}{l}37.4 \\
(52 / 139)\end{array}$ & $\begin{array}{l}28.1 \\
(39 / 139)\end{array}$ & $\begin{array}{l}1.4 \\
(2 / 139)\end{array}$ & $\begin{array}{l}25.9 \\
(36 / 139)\end{array}$ & $\begin{array}{l}3.6 \\
(5 / 139)\end{array}$ \\
\hline Hungary & 120 & $\begin{array}{l}0.0 \\
(0 / 120)\end{array}$ & $\begin{array}{l}8.3 \\
(10 / 120)\end{array}$ & $\begin{array}{l}25.8 \\
(1 / 120)\end{array}$ & $\begin{array}{l}28.3 \\
(34 / 120)\end{array}$ & $\begin{array}{l}23.3 \\
(28 / 120)\end{array}$ & $\begin{array}{l}10.0 \\
(12 / 120)\end{array}$ & $\begin{array}{l}0.8 \\
(1 / 120)\end{array}$ & $\begin{array}{l}3.3 \\
(4 / 120)\end{array}$ \\
\hline Portugal & 157 & $\begin{array}{l}1.3 \\
(2 / 157)\end{array}$ & $\begin{array}{l}23.6 \\
(37 / 157)\end{array}$ & $\begin{array}{l}38.2 \\
(60 / 157)\end{array}$ & $\begin{array}{l}15.9 \\
(25 / 157)\end{array}$ & $\begin{array}{l}8.9 \\
(14 / 157)\end{array}$ & $\begin{array}{l}5.1 \\
(8 / 157)\end{array}$ & $\begin{array}{l}3.8 \\
(6 / 157)\end{array}$ & $\begin{array}{l}3.2 \\
(5 / 157)\end{array}$ \\
\hline Romania & 169 & $\begin{array}{l}1.2 \\
(2 / 168)\end{array}$ & $\begin{array}{l}4.2 \\
(7 / 168)\end{array}$ & $\begin{array}{l}21.4 \\
(36 / 168)\end{array}$ & $\begin{array}{l}14.3 \\
(24 / 168)\end{array}$ & $\begin{array}{l}17.3 \\
(29 / 168)\end{array}$ & $\begin{array}{l}1.2 \\
(2 / 168)\end{array}$ & $\begin{array}{l}10.1 \\
(17 / 168)\end{array}$ & $\begin{array}{l}32.4^{\mathrm{d}} \\
(51 / 168)\end{array}$ \\
\hline Russia & 151 & $\begin{array}{l}0.0 \\
(0 / 151)\end{array}$ & $\begin{array}{l}13.2 \\
(20 / 151)\end{array}$ & $\begin{array}{l}25.8 \\
(39 / 151)\end{array}$ & $\begin{array}{l}18.5 \\
(28 / 151)\end{array}$ & $\begin{array}{l}35.8 \\
(54 / 151)\end{array}$ & $\begin{array}{l}4.0 \\
(6 / 151)\end{array}$ & $\begin{array}{l}0.0 \\
(0 / 151)\end{array}$ & $\begin{array}{l}2.6 \\
(4 / 151)\end{array}$ \\
\hline Spain & 38 & $\begin{array}{l}0.0 \\
(0 / 38)\end{array}$ & $\begin{array}{l}13.2 \\
(5 / 38)\end{array}$ & $\begin{array}{l}26.3 \\
(10 / 38)\end{array}$ & $\begin{array}{l}31.6 \\
(12 / 38)\end{array}$ & $\begin{array}{l}10.5 \\
(4 / 38)\end{array}$ & $\begin{array}{l}15.8 \\
(6 / 38)\end{array}$ & $\begin{array}{l}2.6 \\
(1 / 38)\end{array}$ & $\begin{array}{l}0.0 \\
(0 / 38)\end{array}$ \\
\hline Switzerland & 54 & $\begin{array}{l}4.2 \\
(2 / 48)\end{array}$ & $\begin{array}{l}33.3 \\
(16 / 48)\end{array}$ & $\begin{array}{l}41.7 \\
(20 / 48)\end{array}$ & $\begin{array}{l}0.0 \\
(0 / 48)\end{array}$ & $\begin{array}{l}6.3 \\
(3 / 48)\end{array}$ & $\begin{array}{l}6.3 \\
(3 / 48)\end{array}$ & $\begin{array}{l}2.1 \\
(1 / 48)\end{array}$ & $\begin{array}{l}6.3 \\
(3 / 48)\end{array}$ \\
\hline
\end{tabular}

${ }^{\text {a }}$ Percentages of patients being evaluated.

${ }^{\mathrm{b}}$ Number of patients with the index diagnosis versus total numbers of patients being evaluated (compare also with the total number of patients being recruited, left column).

${ }^{c}$ Includes patients with ICD-system, pacemaker, stable angina.

${ }^{\mathrm{d}}$ Romania: $23.8 \%$ patients with stable angina; yellow background = referral diagnosis represents 10 -30\%; grey background = above $30 \%$.

was possible only at the sites, where patients originally were enrolled to enable additional information if needed. The Institut für Herzinfarktforschung (IHF) Ludwigshafen, Germany provided overall data management, statistical analysis and site-specific reports.

All collected data were offered to the national coordinators for benchmarking and quality control within the country where the data have been collected. Benchmarking of individual centres' data with the pooled data of the other centres was possible after permission by the EuroCaReD steering committee and the national coordinators. No data from any individual centre were released to other centres.

\subsection{Statistical analysis}

Continuous variables are presented as means with standard deviations medians with 25 th and 75 th percentiles and were compared by using Wilcoxon test. Categorical variables and completeness of documentation (data availability) are presented as absolute numbers or percentages and were compared by using chi-square test. The statistical comparisons were two-tailed, and p-values $<0.05$ were considered as statistically significant. For identifying independent predictors of drop-out from CR a logistic regression analysis was done. All analyses were performed using SAS version 9.3 (SAS Institute Inc., Cary, NC, USA).

\subsection{Ethical review board approval}

The national coordinators of the EuroCaReD project were responsible for getting approval of the national or local ethical committees, according to the prevailing national requirements. 
Table 2

Demographic characteristics and cardiovascular risk factors in history of patients referred to CR.

\begin{tabular}{|c|c|c|c|c|c|c|c|c|c|c|}
\hline Country & $\begin{array}{l}\text { Total } \\
\text { number of } \\
\text { patients }\end{array}$ & $\begin{array}{l}\text { Age (years) }{ }^{\mathrm{a}} \\
\text { female }(\%)^{\mathrm{a}}\end{array}$ & $\begin{array}{l}\text { Retired } \\
(\%)(\text { DA \% })^{b}\end{array}$ & $\begin{array}{l}\text { Hyper-tension } \\
(\%)(\text { DA \% })^{\mathrm{b}}\end{array}$ & $\begin{array}{l}\text { Elevated } \\
\text { LDL-cholesterol } \\
(\%)(\mathrm{DA} \%)^{\mathrm{b}}\end{array}$ & $\begin{array}{l}\text { Diabetes } \\
\text { mellitus (\%) } \\
(\mathrm{DA} \%)^{\mathrm{b}}\end{array}$ & $\begin{array}{l}\text { Overweight } \\
\text { obesity (\%) } \\
(\mathrm{DA} \%)^{\mathrm{b}}\end{array}$ & $\begin{array}{l}\text { Smoking } \\
(\%)(\mathrm{DA} \%)^{\mathrm{b}}\end{array}$ & $\begin{array}{l}\text { Low/no physical } \\
\text { activity (\%) (DA \%) }\end{array}$ & $\begin{array}{l}\text { Depression } \\
(\%)(\mathrm{DA} \%)^{\mathrm{b}}\end{array}$ \\
\hline Austria & 544 & $\begin{array}{l}59.0 \\
19.7\end{array}$ & $\begin{array}{l}50.7 \\
(94)\end{array}$ & $\begin{array}{l}50.7 \\
(92)\end{array}$ & $\begin{array}{l}59.6 \\
(92)\end{array}$ & $\begin{array}{l}13.1 \\
(92)\end{array}$ & $\begin{array}{l}29.0 \\
(92)\end{array}$ & $\begin{array}{l}54.7 \\
(33)\end{array}$ & $\begin{array}{l}29.0 \\
(92)\end{array}$ & NA \\
\hline Belgium & 232 & $\begin{array}{l}62.5 \\
23.3\end{array}$ & $\begin{array}{l}51.4 \\
(60)\end{array}$ & $\begin{array}{l}47.7 \\
(92)\end{array}$ & $\begin{array}{l}66.8 \\
(90)\end{array}$ & $\begin{array}{l}19.2 \\
(92)\end{array}$ & $\begin{array}{l}36.9 \\
(89)\end{array}$ & $\begin{array}{l}29.0 \\
(92)\end{array}$ & $\begin{array}{l}55.6 \\
(84)\end{array}$ & $\begin{array}{l}11.6 \\
(75)\end{array}$ \\
\hline Croatia & 93 & $\begin{array}{l}62.0 \\
23.7\end{array}$ & $\begin{array}{l}59.1 \\
(100)\end{array}$ & $\begin{array}{l}86.0 \\
(100)\end{array}$ & $\begin{array}{l}94.6 \\
(99)\end{array}$ & $\begin{array}{l}34.8 \\
(99)\end{array}$ & $\begin{array}{l}35.5 \\
(100)\end{array}$ & $\begin{array}{l}31.2 \\
(100)\end{array}$ & $\begin{array}{l}55.9 \\
(100)\end{array}$ & $\begin{array}{l}1.1 \\
(75)\end{array}$ \\
\hline Croatia & 153 & $\begin{array}{l}61.0 \\
24.8\end{array}$ & $\begin{array}{l}52.1 \\
(86)\end{array}$ & $\begin{array}{l}52.1 \\
(94)\end{array}$ & $\begin{array}{l}86.1 \\
(94)\end{array}$ & $\begin{array}{l}22 \\
(95)\end{array}$ & $\begin{array}{l}44.7 \\
(92)\end{array}$ & $\begin{array}{l}47.6 \\
(93)\end{array}$ & $\begin{array}{l}46.5 \\
(54)\end{array}$ & $\begin{array}{l}7.6 \\
(86)\end{array}$ \\
\hline Germany & 204 & $\begin{array}{l}58.0 \\
20.6\end{array}$ & $\begin{array}{l}36.2 \\
(99)\end{array}$ & $\begin{array}{l}72.3 \\
(99)\end{array}$ & $\begin{array}{l}88.0 \\
(98)\end{array}$ & $\begin{array}{l}23.6 \\
(99)\end{array}$ & $\begin{array}{l}44.1 \\
(100)\end{array}$ & $\begin{array}{l}38.4 \\
(99)\end{array}$ & $\begin{array}{l}62.0 \\
(84)\end{array}$ & $\begin{array}{l}13.2 \\
(93)\end{array}$ \\
\hline Greece & 139 & $\begin{array}{l}65.0 \\
30.2\end{array}$ & $\begin{array}{l}72.8 \\
(98)\end{array}$ & $\begin{array}{l}84.1 \\
(99)\end{array}$ & $\begin{array}{l}71.9 \\
(100)\end{array}$ & $\begin{array}{l}23.7 \\
(100)\end{array}$ & $\begin{array}{l}16.5 \\
(100)\end{array}$ & $\begin{array}{l}33.8 \\
(98)\end{array}$ & $\begin{array}{l}80.3 \\
(95)\end{array}$ & $\begin{array}{l}3.6 \\
(99)\end{array}$ \\
\hline Hungary & 120 & $\begin{array}{l}60.0 \\
24.2\end{array}$ & $\begin{array}{l}52.5 \\
(100)\end{array}$ & $\begin{array}{l}85.7 \\
(99)\end{array}$ & $\begin{array}{l}73.9 \\
(99)\end{array}$ & $\begin{array}{l}29.7 \\
(99)\end{array}$ & $\begin{array}{l}43.7 \\
(99)\end{array}$ & $\begin{array}{l}55.9 \\
(99)\end{array}$ & $\begin{array}{l}73.9 \\
(99)\end{array}$ & $\begin{array}{l}3.4 \\
(98)\end{array}$ \\
\hline Portugal & 157 & $\begin{array}{l}59.0 \\
19.7\end{array}$ & $\begin{array}{l}35.5 \\
(99)\end{array}$ & $\begin{array}{l}55.8 \\
(99)\end{array}$ & $\begin{array}{l}69.0 \\
(99)\end{array}$ & $\begin{array}{l}24.3 \\
(97)\end{array}$ & $\begin{array}{l}34.0 \\
(97)\end{array}$ & $\begin{array}{l}35.9 \\
(99)\end{array}$ & $\begin{array}{l}83.8 \\
(99)\end{array}$ & $\begin{array}{l}16 \\
(99)\end{array}$ \\
\hline Romania & 169 & $\begin{array}{l}62.0 \\
42.6\end{array}$ & $\begin{array}{l}62.8 \\
(81)\end{array}$ & $\begin{array}{l}78.1 \\
(100)\end{array}$ & $\begin{array}{l}58.0 \\
(100)\end{array}$ & $\begin{array}{l}25.4 \\
(100)\end{array}$ & $\begin{array}{l}42.0 \\
(100)\end{array}$ & $\begin{array}{l}32.1 \\
(98)\end{array}$ & $\begin{array}{l}72.3 \\
(88)\end{array}$ & $\begin{array}{l}17.6 \\
(88)\end{array}$ \\
\hline Russia & 151 & $\begin{array}{l}57.0 \\
23.8\end{array}$ & $\begin{array}{l}29.3 \\
(99)\end{array}$ & $\begin{array}{l}84.1 \\
(100)\end{array}$ & $\begin{array}{l}60.7 \\
(99)\end{array}$ & $\begin{array}{l}15.9 \\
(100)\end{array}$ & $\begin{array}{l}47.3 \\
(99)\end{array}$ & $\begin{array}{l}43.3 \\
(99)\end{array}$ & $\begin{array}{l}43 \\
(99)\end{array}$ & $\begin{array}{l}9 \\
(95)\end{array}$ \\
\hline Spain & 38 & $\begin{array}{l}57.0 \\
10.5\end{array}$ & $\begin{array}{l}34.8 \\
(61)\end{array}$ & $\begin{array}{l}50.0 \\
(100)\end{array}$ & $\begin{array}{l}57.9 \\
(100)\end{array}$ & $\begin{array}{l}35.1 \\
(99)\end{array}$ & $\begin{array}{l}40.5 \\
(99)\end{array}$ & $\begin{array}{l}44.4 \\
(99)\end{array}$ & $\begin{array}{l}63.2 \\
(100)\end{array}$ & $\begin{array}{l}11.1 \\
(99)\end{array}$ \\
\hline Switzerland & 54 & $\begin{array}{l}60.5 \\
22.2\end{array}$ & $\begin{array}{l}61 \\
(78)\end{array}$ & $\begin{array}{l}63.0 \\
(100)\end{array}$ & $\begin{array}{l}51.9 \\
(100)\end{array}$ & $\begin{array}{l}16.7 \\
(100)\end{array}$ & $\begin{array}{l}29.6 \\
(100)\end{array}$ & $\begin{array}{l}52.8 \\
(99)\end{array}$ & $\begin{array}{l}42.3 \\
(98)\end{array}$ & $\begin{array}{l}7.4 \\
(100)\end{array}$ \\
\hline Total population & 2054 & $\begin{array}{l}61.0 \\
23.8\end{array}$ & $\begin{array}{l}48.7 \\
(90.0)\end{array}$ & $\begin{array}{l}64.4 \\
(96.4)\end{array}$ & $\begin{array}{l}69.0 \\
(96.0)\end{array}$ & $\begin{array}{l}16.9 \\
(96.1)\end{array}$ & $\begin{array}{l}36.0 \\
(95.8)\end{array}$ & $\begin{array}{l}32.4 \\
(80.1)\end{array}$ & $\begin{array}{l}53.7 \\
(91.4)\end{array}$ & $\begin{array}{l}9.8 \\
(67.8)\end{array}$ \\
\hline
\end{tabular}

a Median.

b DA, data availability defined as \% of patients of the study population being evaluated for the individual item under consideration.

\section{Results}

\subsection{Study population}

Primarily 2.095 patients from 71 CR centres in 14 European countries have been enrolled during the predefined study periods. After examination for minimal requirements of data completeness two countries were excluded. Thereby data from 2.054 patients from $69 \mathrm{CR}$ centres of 12 European countries were suitable for the final analysis. The numbers of patients being enrolled in each country are given in Tables 1-4.

\subsection{Clinical events initiating $C R$}

Regarding the entire study population, patients with coronary artery disease (CAD) represented the vast majority of CR patients (83\%). The most common initiating events for CR referral were acute coronary syndrome (ACS) (40\%) and elective PCI (22\%), followed by patients after CABG (19\%) (Table 1). The number of patients admitted for $\mathrm{CR}$ because of chronic heart failure (CHF) was low (4\%). The most relevant comorbidities were musculoskeletal disorders (18\%) and COPD (11\%).

Table 3

Patients prescribed medication at CR programme start.

\begin{tabular}{|c|c|c|c|c|c|c|c|c|c|}
\hline \multirow[b]{2}{*}{ Country } & \multirow[b]{2}{*}{$\mathrm{N}$} & \multicolumn{8}{|c|}{ Medication as applied in \% of patients evaluated (patients treated/patients evaluated) } \\
\hline & & ASS & $\mathrm{TP}$ & BB & ACE-I & ARB & Statin & Oral diabetes control & Insulin \\
\hline Austria & 544 & $\begin{array}{l}81 \\
(29)\end{array}$ & $\begin{array}{l}64.6 \\
(29)\end{array}$ & $\begin{array}{l}80.4 \\
(29)\end{array}$ & $\begin{array}{l}49.4 \\
(29)\end{array}$ & NA & $\begin{array}{l}81.6 \\
(29)\end{array}$ & $\begin{array}{l}9.5 \\
(29)\end{array}$ & $\begin{array}{l}2.5 \\
(29)\end{array}$ \\
\hline Belgium & 232 & $\begin{array}{l}91 \\
(63)\end{array}$ & $\begin{array}{l}36.4 \\
(62)\end{array}$ & $\begin{array}{l}85 \\
(63)\end{array}$ & $\begin{array}{l}47.2 \\
(61)\end{array}$ & $\begin{array}{l}5.9 \\
(59)\end{array}$ & $\begin{array}{l}81.4 \\
(63)\end{array}$ & $\begin{array}{l}16.1 \\
(62)\end{array}$ & $\begin{array}{l}4.2 \\
(62)\end{array}$ \\
\hline Croatia & 93 & $\begin{array}{l}83.7 \\
(99)\end{array}$ & $\begin{array}{l}58.7 \\
(99)\end{array}$ & $\begin{array}{l}96.7 \\
(99)\end{array}$ & $\begin{array}{l}65.2 \\
(99)\end{array}$ & $\begin{array}{l}19.8 \\
(98)\end{array}$ & $\begin{array}{l}91.3 \\
(99)\end{array}$ & $\begin{array}{l}23.3 \\
(97)\end{array}$ & $\begin{array}{l}3.3 \\
(98)\end{array}$ \\
\hline Denmark & 153 & $\begin{array}{l}92.9 \\
79 / 85\end{array}$ & $\begin{array}{l}64.2 \\
43 / 67\end{array}$ & $\begin{array}{l}79.2 \\
61 / 77\end{array}$ & $\begin{array}{l}44.3 \\
31 / 70\end{array}$ & $\begin{array}{l}17.2 \\
11 / 64\end{array}$ & $\begin{array}{l}91.9 \\
79 / 86\end{array}$ & $\begin{array}{l}19.7 \\
13 / 66\end{array}$ & $\begin{array}{l}9.4 \\
6 / 64\end{array}$ \\
\hline Germany & 204 & $\begin{array}{l}83.7 \\
(99)\end{array}$ & $\begin{array}{l}56.7 \\
(98)\end{array}$ & $\begin{array}{l}86.1 \\
(99)\end{array}$ & $\begin{array}{l}63.9 \\
(99)\end{array}$ & $\begin{array}{l}17.8 \\
(99)\end{array}$ & $\begin{array}{l}81.2 \\
(99)\end{array}$ & $\begin{array}{l}15.5 \\
(98)\end{array}$ & $\begin{array}{l}9.5 \\
(99)\end{array}$ \\
\hline Greece & 139 & $\begin{array}{l}80 \\
96 / 120\end{array}$ & $\begin{array}{l}31.4 \\
38 / 121\end{array}$ & $\begin{array}{l}93.4 \\
113 / 121\end{array}$ & $\begin{array}{l}86 \\
104 / 121\end{array}$ & $\begin{array}{l}14.2 \\
17 / 120\end{array}$ & $\begin{array}{l}93.4 \\
113 / 121\end{array}$ & $\begin{array}{l}16.5 \\
20 / 121\end{array}$ & $\begin{array}{l}2.5 \\
3 / 121\end{array}$ \\
\hline Hungary & 120 & $\begin{array}{l}89.2 \\
107 / 120\end{array}$ & $\begin{array}{l}62.5 \\
75 / 120\end{array}$ & $\begin{array}{l}97.5 \\
117 / 120\end{array}$ & $\begin{array}{l}75.8 \\
91 / 120\end{array}$ & $\begin{array}{l}16.8 \\
20 / 119\end{array}$ & $\begin{array}{l}87.5 \\
105 / 120\end{array}$ & $\begin{array}{l}21.8 \\
26 / 119\end{array}$ & $\begin{array}{l}7.6 \\
9 / 119\end{array}$ \\
\hline Portugal & 157 & $\begin{array}{l}95 \\
148 / 155\end{array}$ & $\begin{array}{l}87.2 \\
136 / 156\end{array}$ & $\begin{array}{l}86.3 \\
132 / 153\end{array}$ & $\begin{array}{l}72.9 \\
113 / 155\end{array}$ & $\begin{array}{l}12.9 \\
20 / 155\end{array}$ & $\begin{array}{l}94.2 \\
146 / 155\end{array}$ & $\begin{array}{l}21.3 \\
33 / 155\end{array}$ & $\begin{array}{l}8.4 \\
13 / 155\end{array}$ \\
\hline Romania & 169 & $\begin{array}{l}84.7 \\
(116 / 137)\end{array}$ & $\begin{array}{l}46 \\
(63 / 137)\end{array}$ & $\begin{array}{l}88.3 \\
(121 / 137)\end{array}$ & $\begin{array}{l}73 \\
(100 / 137)\end{array}$ & $\begin{array}{l}14 \\
(19 / 136)\end{array}$ & $\begin{array}{l}85.3 \\
(116 / 136)\end{array}$ & $\begin{array}{l}17.6 \\
(24 / 136)\end{array}$ & $\begin{array}{l}2.2 \\
(3 / 137)\end{array}$ \\
\hline Russia & 151 & $\begin{array}{l}94.5 \\
(137 / 145)\end{array}$ & $\begin{array}{l}51 \\
(74 / 145)\end{array}$ & $\begin{array}{l}91.7 \\
(133 / 145)\end{array}$ & $\begin{array}{l}77.2 \\
(112 / 145)\end{array}$ & $\begin{array}{l}5.5 \\
(8 / 145)\end{array}$ & $\begin{array}{l}93.8 \\
(136 / 145)\end{array}$ & $\begin{array}{l}9.7 \\
(14 / 145)\end{array}$ & $\begin{array}{l}0.0 \\
(0 / 145)\end{array}$ \\
\hline Spain & 38 & $\begin{array}{l}94.4 \\
(17 / 18)\end{array}$ & $\begin{array}{l}44.4 \\
(8 / 18)\end{array}$ & $\begin{array}{l}77.8 \\
(14 / 18)\end{array}$ & $\begin{array}{l}55.6 \\
(10 / 18)\end{array}$ & $\begin{array}{l}16.7 \\
(3 / 18)\end{array}$ & $\begin{array}{l}100 \\
(18 / 18)\end{array}$ & $\begin{array}{l}22.2 \\
(4 / 18)\end{array}$ & $\begin{array}{l}0.0 \\
(0.18)\end{array}$ \\
\hline Switzerland & 54 & $\begin{array}{l}81.4 \\
(35 / 43)\end{array}$ & $\begin{array}{l}81.4 \\
(35 / 43)\end{array}$ & $\begin{array}{l}88.4 \\
(38 / 43)\end{array}$ & $\begin{array}{l}65.1 \\
(28 / 43)\end{array}$ & $\begin{array}{l}25.6 \\
(11 / 43)\end{array}$ & $\begin{array}{l}88.4 \\
(38 / 43)\end{array}$ & $\begin{array}{l}4.7 \\
(2 / 43)\end{array}$ & $\begin{array}{l}9.5 \\
(4 / 42)\end{array}$ \\
\hline
\end{tabular}


The distribution of initiating events exhibited a considerable heterogeneity between the participating countries. Whereas ACS dominated in Switzerland (79\%), Portugal (62\%) and Germany (61\%), elective PCI for stable CAD was prominent in Greece (37\%), Austria (36\%) and Spain (32\%). Only in Switzerland CR referral of patients with NSTEMI (33\%) was comparable to the group of patients with STEMI, whereas in some countries NSTEMI patients were not represented at all within this survey (e.g. Greece). Patients after CABG represented the largest group in Croatia and Russia (36\% each).

\subsection{Demographic characteristics and history of CV risk factors}

The mean age of the total population was $58 \pm 16$ years with low variations between the countries. $76 \%$ of the study population were male. Female patients represented the minority in all countries and ranged from $10 \%$ in Spain to $43 \%$ in Romania. At CR start almost half of the patients were retired (49\%) with a maximum in Greece (73\%) and a minimum in Russia (29\%).

Most patients had a typical CV risk profile with a history of hyperlipidaemia (69\%), hypertension (64\%), lack of regular physical activity (54\%) and smoking (40\%). However, the distribution of the individual risk factors in patients' history considerably varied between countries. Percentages were displayed in Table 2. The distribution was in hypertension from 48\% (Belgium) to 84\% (Russia); in hyperlipidaemia from 52\% (Switzerland) to 95\% (Croatia); in diabetes mellitus from 13\% (Austria) to 35\% (Spain); in overweight/obesity form $16 \%$ (Greece) to $47 \%$ (Russia); in smoking from $29 \%$ (Belgium) to $53 \%$ Switzerland; in low/no physical activity from 29\% (Austria) to $84 \%$ (Portugal); and in depression from $1 \%$ (Croatia) to $18 \%$ (Romania).
3.4. Baseline hemodynamic parameters and medication prescription in patients referred to $C R$

The large majority of patients were in sinus rhythm. At CR start mean heart rate at rest was $69 \mathrm{bpm}$ and mean left ventricular ejection fraction (LVEF) 54\%. All patients were on guideline-adjusted medication before starting the CR programme (Table 3 ).

\subsection{Changes of individual risk factors during CR programme}

Table 4 presents CV risk factor data at CR start and end of those patients with the individual risk factors reported in history (Table 2). With respect to blood pressure, LDL-cholesterol and baseline glucose, patients were already on medication at CR start but the values still could be optimized during CR (values at CR start and change as measured at CR end: systolic blood pressure $131=$ minus $5 \mathrm{~mm} \mathrm{Hg}$; LDLcholesterol: $108 \mathrm{mg} / \mathrm{dl}=-18 \mathrm{mg} / \mathrm{dl}$; and fasting glucose $136 \mathrm{mg} / \mathrm{dl}=-11 \mathrm{mg} / \mathrm{dl}$. Notably, baseline measurements of these risk factors were incomplete, and controls were performed even less. Mean BMI of patients with overweight or obesity in history was $32 \mathrm{~kg} / \mathrm{m}^{2}$, and a relevant reduction could not be achieved during CR. From the group of patients with history of smoking a relevant part already stopped before $\mathrm{CR}$ start, however, this part showed a large variation between the countries (Table 4). Unfortunately the further course of smoking behaviour has not been reported by the CR centres. Therefore the effect of CR on smoking behaviour could not be assessed.

\subsection{Exercise capacity and CR success}

Exercise capacity given in "watts" could be documented only in 535 patients ( $28 \%$ of total), and an even lower number was documented at

Table. 4

Subgroups of patients with individual cardiovascular risk factors in history: evaluation at CR start and changes during CR.

\begin{tabular}{|c|c|c|c|c|c|c|c|c|}
\hline Country & $\begin{array}{l}\text { Total } \\
\text { number } \\
\text { of } \\
\text { patients } \\
\text { recruited }\end{array}$ & $\begin{array}{l}\text { Syst. blood pressure } \\
\text { (patients with } \\
\text { hypertension in } \\
\text { history only) } \\
\text { change }^{\mathrm{a}}(\mathrm{mm} \mathrm{Hg}) \\
\text { [patients } \\
\text { investigated] }\end{array}$ & $\begin{array}{l}\text { LDL-cholesterol } \\
\text { (patients with } \\
\text { hyperlipidaemia } \\
\text { in } \\
\text { history only) }^{\mathrm{a}} \\
\text { change }^{\mathrm{a}}(\mathrm{mg} / \mathrm{dl} \text { ) } \\
\text { [patients }_{\text {investigated] }}\end{array}$ & $\begin{array}{l}\text { Baseline glucose } \\
\text { (patients with } \\
\text { diabetes in history } \\
\text { only) }^{\mathrm{a}} \text { change } \\
(\mathrm{mg} / \mathrm{dl}) \text { [patients } \\
\text { investigated] }\end{array}$ & $\begin{array}{l}\text { Body mass index } \\
\text { (patients with } \\
\text { overweight in } \\
\text { history only) } \\
\text { change }^{\mathrm{a}}\left(\mathrm{kg} / \mathrm{m}^{2}\right) \\
\text { [patients } \\
\text { investigated] }\end{array}$ & $\begin{array}{l}\text { Current } \\
\text { smokers in } \\
\text { history }^{\mathrm{a}} \text { at CR } \\
\text { start }^{\mathrm{ab}}(\%) \\
\text { [patients } \\
\text { investigated] }\end{array}$ & $\begin{array}{l}\text { Exercise } \\
\text { capacity } \\
\text { (watts, all } \\
\text { patients) } \\
\text { change } \\
\text { (watts) } \\
\text { [patients } \\
\text { investigated] }\end{array}$ & $\begin{array}{l}\text { Exercise capacity } \\
\text { (watts, patients with } \\
\text { "no physical activity in } \\
\text { history" only) change } \\
\text { (watts) [patients } \\
\text { investigated] }\end{array}$ \\
\hline Austria & 544 & $\begin{array}{l}128.8[243] \\
-3.8[165]\end{array}$ & $\begin{array}{l}104.2[275] \\
-17.6[197]\end{array}$ & $\begin{array}{l}140.9[42] \\
-4.9[34]\end{array}$ & $\begin{array}{l}31.3[144] \\
-0.6[101]\end{array}$ & $\begin{array}{l}54.7[181] \\
50.0[178]\end{array}$ & $\begin{array}{l}143[40] \\
+28[38]\end{array}$ & $\begin{array}{l}96[4] \\
+19[2]\end{array}$ \\
\hline Belgium & 232 & $\begin{array}{l}138.3[68] \\
-2.5[39]\end{array}$ & $\begin{array}{l}104.4[71] \\
-34.2[29]\end{array}$ & $\begin{array}{l}126.9[15] \\
-29.3[3]\end{array}$ & $\begin{array}{l}30.9 \\
-0.2[0.2]\end{array}$ & $\begin{array}{l}29.0[214] \\
19.9[146]\end{array}$ & $\begin{array}{l}112[111] \\
+22[66]\end{array}$ & $\begin{array}{l}100[53] \\
+17[29]\end{array}$ \\
\hline Croatia & 93 & $\begin{array}{l}132.0[80] \\
-10.9[78]\end{array}$ & $\begin{array}{l}87.6[87] \\
-7.0[29]\end{array}$ & $\begin{array}{l}145.5[31] \\
-17.2[29]\end{array}$ & $\begin{array}{l}33.9[32] \\
-0.5[24]\end{array}$ & $\begin{array}{l}31.2[93] \\
31.2[93]\end{array}$ & $\begin{array}{l}106[62] \\
+13[15]\end{array}$ & $\begin{array}{l}94[31] \\
+18[10]\end{array}$ \\
\hline Denmark & 153 & $\begin{array}{l}138.2[67] \\
+1.4[37]\end{array}$ & $\begin{array}{l}100.0[110] \\
-24.2[71]\end{array}$ & $\begin{array}{l}130.6[23] \\
-13.6[9]\end{array}$ & $\begin{array}{l}30.9[54] \\
+0.1[31]\end{array}$ & $\begin{array}{l}47.6[143] \\
22.2[135]\end{array}$ & $\begin{array}{l}114[1] \\
\mathrm{NA}^{\mathrm{c}}\end{array}$ & $N A^{c}$ \\
\hline Germany & 204 & $\begin{array}{l}129.7[145] \\
-5.9[141]\end{array}$ & $\begin{array}{l}107.7[165] \\
-17.9[136]\end{array}$ & $\begin{array}{l}135.9[43] \\
-14.1[39]\end{array}$ & $\begin{array}{l}32.5[88] \\
-0.7[83]\end{array}$ & $\begin{array}{l}38.4[203] \\
17.2[204]\end{array}$ & $\begin{array}{l}98[188] \\
+21[148]\end{array}$ & $\begin{array}{l}95[96] \\
+17[75]\end{array}$ \\
\hline Greece & 139 & $\begin{array}{l}117.9[109] \\
+0.03[104]\end{array}$ & $\begin{array}{l}111.4[94] \\
-1.5[89]\end{array}$ & $\begin{array}{l}117.8[28] \\
-1.1[25]\end{array}$ & $\begin{array}{l}33.8[23] \\
-0.3[18]\end{array}$ & $\begin{array}{l}33.8[136] \\
13.0[138]\end{array}$ & $\begin{array}{l}106[11] \\
+1[1]\end{array}$ & $\begin{array}{l}65[3] \\
+1[1]\end{array}$ \\
\hline Hungary & 120 & $\begin{array}{l}133.3[102] \\
-9.3[102]\end{array}$ & $\begin{array}{l}110.0[54] \\
-16.0[24]\end{array}$ & $\begin{array}{l}139.9[27] \\
-5.5[25]\end{array}$ & $\begin{array}{l}32.6[50] \\
-0.5[49]\end{array}$ & $\begin{array}{l}55.9[118] \\
10.0[120]\end{array}$ & $\begin{array}{l}109[8] \\
N A^{c}\end{array}$ & $\begin{array}{l}109[8] \\
N A^{\mathrm{C}}\end{array}$ \\
\hline Portugal & 157 & $\begin{array}{l}133.2[86] \\
-7.9[73]\end{array}$ & $\begin{array}{l}112.9[94] \\
-22.6[75]\end{array}$ & $\begin{array}{l}154.4[33] \\
-21.0[28]\end{array}$ & $\begin{array}{l}31.3[52] \\
-1.2[47]\end{array}$ & $\begin{array}{l}35.9[156] \\
23.4[154]\end{array}$ & $N A^{c}$ & $N A^{c}$ \\
\hline Romania & 169 & $\begin{array}{l}138.5[103] \\
-8.9[74]\end{array}$ & $\begin{array}{l}141.9[70] \\
-38.9[48]\end{array}$ & $\begin{array}{l}139.3[34] \\
-12.7[23]\end{array}$ & $\begin{array}{l}33.5[60] \\
-0.7[45]\end{array}$ & $\begin{array}{l}32.1[165] \\
21.9[137]\end{array}$ & $\begin{array}{l}74[62] \\
+41[29]\end{array}$ & $\begin{array}{l}69[40] \\
+44[17]\end{array}$ \\
\hline Russia & 151 & $\begin{array}{l}129.4[127] \\
-8.6[120]\end{array}$ & $\begin{array}{l}123.2[69] \\
-13.4[13]\end{array}$ & $\begin{array}{l}117.0[24] \\
-18.8[19]\end{array}$ & $\begin{array}{l}31.4[71] \\
+0.2[67]\end{array}$ & $\begin{array}{l}43.3[150] \\
39.3[150]\end{array}$ & $\mathrm{NA}^{\mathrm{c}}$ & $N A^{c}$ \\
\hline Spain & 38 & $\begin{array}{l}128.9[9] \\
-12.5[8]\end{array}$ & $\begin{array}{l}91.2[13] \\
-15.5[12]\end{array}$ & $\begin{array}{l}109.1[7] \\
-3.4[5]\end{array}$ & $\begin{array}{l}27.7[8] \\
-2.1[7]\end{array}$ & $\begin{array}{l}44.4[36] \\
20.8[24]\end{array}$ & $\begin{array}{l}N A^{c} \\
N A^{c}\end{array}$ & $N^{c}$ \\
\hline Switzer land & 54 & $\begin{array}{l}125.7[34] \\
+6.8[26]\end{array}$ & $\begin{array}{l}92.9[13] \\
N A^{c}\end{array}$ & $\begin{array}{l}142.6[2] \\
N A^{\mathrm{C}}\end{array}$ & $\begin{array}{l}30.6[30.6] \\
0.0[9]\end{array}$ & $\begin{array}{l}52.8[53] \\
13.0[54]\end{array}$ & $\begin{array}{l}113[52] \\
+30[42]\end{array}$ & $\begin{array}{l}102[21] \\
+22[16]\end{array}$ \\
\hline $\begin{array}{l}\text { All } \\
\text { countries }\end{array}$ & 2054 & $\begin{array}{l}130.7[1173] \\
-5.7[967]\end{array}$ & $\begin{array}{l}108.0[1108] \\
-18.3[723]\end{array}$ & $\begin{array}{l}135.7[309] \\
-10.8[239]\end{array}$ & $\begin{array}{l}31.8[652] \\
-0.5[515]\end{array}$ & $\begin{array}{l}40.0[1648] \\
24.7[1533]\end{array}$ & $\begin{array}{l}104[535] \\
+24[339]\end{array}$ & $\begin{array}{l}92[257] \\
+20[150]\end{array}$ \\
\hline
\end{tabular}

\footnotetext{
a Mean values; to increase clarity of presentation standard deviations are not presented.

b Smoking status at the end of CR has not been sufficiently reported, and therefore cannot be presented.

c NA, data not available.
} 


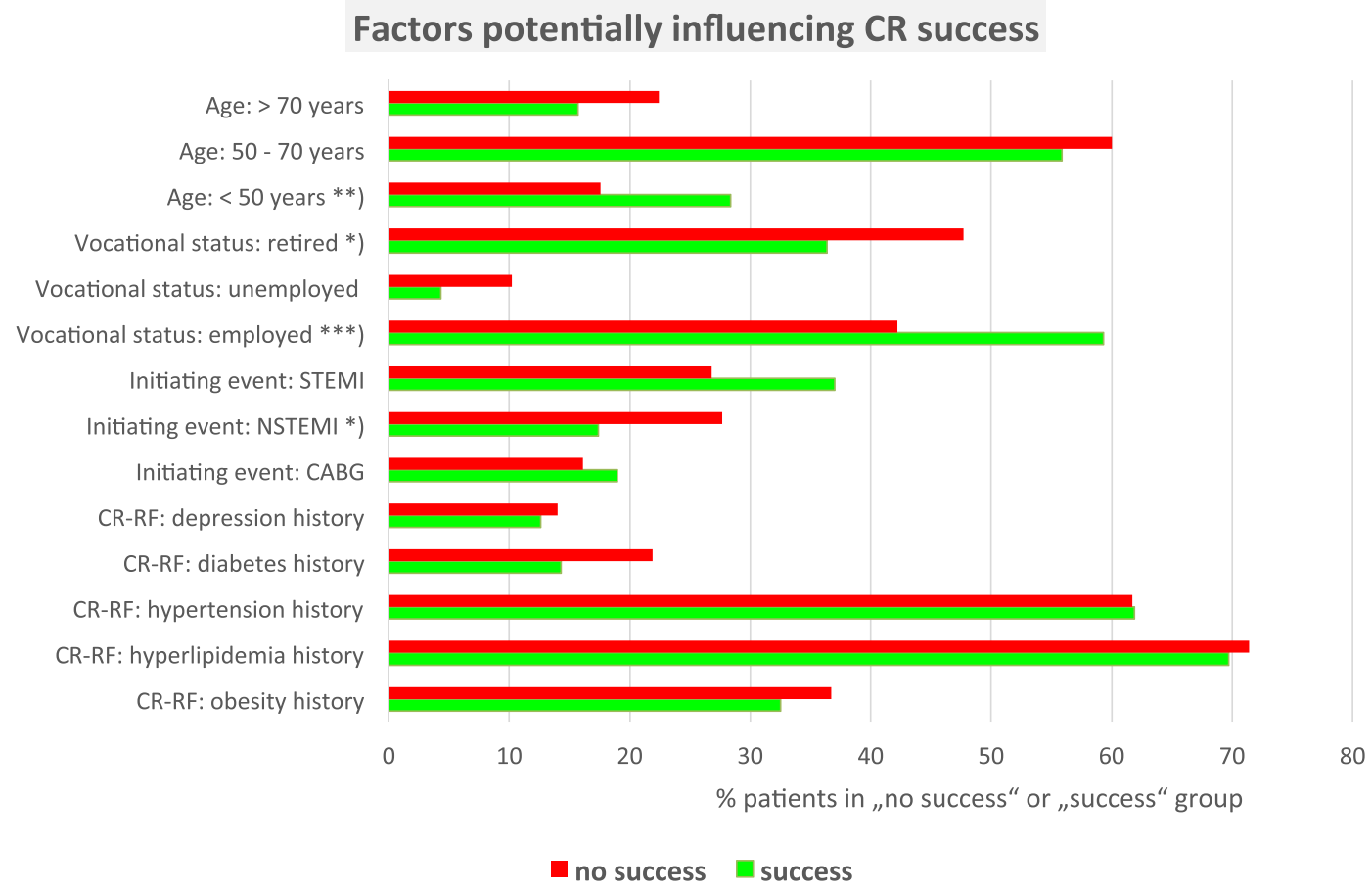

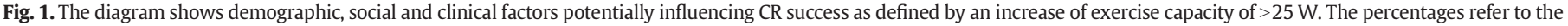

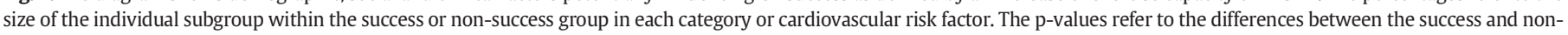
success group; ${ }^{*}$ ) $\left.\mathrm{p}<0.05,{ }^{* *}\right) \mathrm{p}<0.01,{ }^{* * *}$ ) $\mathrm{p}<0.001$

the end of CR too (339; $16 \%$ of total). Therefore repeat bicycle exercise test results at the end of the CR programme were available only in this small subgroup of the study population. Within this subgroup exercise capacity changed from $104 \pm 44 \mathrm{~W}$ at CR start to $128 \pm 50 \mathrm{~W}$ at CR end; $\mathrm{p}<0.0001$ (Table 4 ). Considering only the subgroup with patients' history of "low/no physical activity at CR start" the increase was similar from $92 \pm 38 \mathrm{~W}$ at $\mathrm{CR}$ start to $112 \pm 41 \mathrm{~W}$ at $\mathrm{CR}$ end; $\mathrm{p}<0.0001$ (Table 4).

For further differentiation "CR success" has provisionally been defined as an increase of exercise capacity of $>25 \mathrm{~W}$ after CR. On the basis of this definition 58\% ( $n=198$ ) of the subgroup of patients with repeat exercise tests during $\mathrm{CR}$ successfully completed their programme. The success rate varied according with the patients' baseline characteristics as outlined in Fig. 1. In the subgroup of patients aged $<50$ years, or being employed the number of patients with CR success significantly exceeded the number of unsuccessful patients. In contrast, in the subgroups of patients being retired or patients after NSTEMI the majority of patients remained unsuccessful in increasing their exercise capacity.

\subsection{CR programme characteristics}

CR setting, content and duration varies across countries (Table 5). Mean programme duration was 8 weeks, shortest in Hungary and longest in Greece (24 weeks). During the programmes, an average of 4.2 exercise sessions and 2.8 counselling sessions were offered weekly. The mean total number of sessions offered during CR was 43.5 , highest in Greece $(n=96)$ and lowest in Austria, Denmark, Hungary, and Portugal $(n=30)$.

\section{8. $C R$ programme completion, recurrent $C V$ events and drop-out rate}

Of the 2.054 patients admitted for $\mathrm{CR}, 85 \%$ completed the programme. The most common reasons for patient drop-out were patient non-compliance (31\%), and recurrent CV events within the CR programme timeframe (8\%). But in $60 \%$ of the drop-outs the reason has not been specified.

Table 5

CR setting, duration and content.

\begin{tabular}{|c|c|c|c|c|c|}
\hline Country & Design & Exercise (sessions per week) & Counselling (sessions per week) & Duration (weeks) & Total number of sessions \\
\hline Austria & Outpatient & 3 & 2 & 6 & 30 \\
\hline Belgium & Outpatient & 3 & 1 & 12 & 48 \\
\hline Croatia & Inpatient & 4 & 4 & 4 & 32 \\
\hline Denmark & Outpatient & 3 & 2 & 6 & 30 \\
\hline Germany & In- and outpatient & 12 & 8 & $3(-4)$ & $60(-80)^{\mathrm{a}}$ \\
\hline Greece & Outpatient & 3 & 1 & 24 & 96 \\
\hline Hungary & Inpatient & 5 & 5 & 3 & 30 \\
\hline Portugal & Outpatient & 3 & 2 & 6 & 30 \\
\hline Romania & Inpatient & 4 & 4 & 4 & 32 \\
\hline Russia & Inpatient & 4 & 2 & 6 & 36 \\
\hline Spain & Outpatient & 3 & 1 & 12 & 48 \\
\hline Switzerland & Outpatient & 3 & 2 & 10 & 50 \\
\hline Mean & & 4.2 & 2.8 & 8.0 & 43.5 \\
\hline
\end{tabular}

a Retrospective assessment. 


\subsection{Post-CR disease management}

After CR completion, patients returned to routine care by cardiologists (61\%) and/or to a general practitioner or both (23\%). But only $9 \%$ were admitted to a structured post-CR programme such as "heart group" or equivalent activities.

\subsection{Subgroup analysis for gender}

There was no gender difference in age of CR participants. Fewer women than men were married (70\% vs. $83 \%$; $p<0.0001$ ) but more of them were widowed ( $15 \%$ vs. $4 \% ; \mathrm{p}<0.0001)$. A higher percentage of women were retired ( $54 \%$ vs. $45 \%$; $p<0.001$ ). Significant differences could be observed in the presence of CV risk factors. Fewer women than men reported a history of smoking ( $28 \%$ vs. $44 \%$; $p<0.0001$ ), whereas more women were hypertensive ( $71 \%$ vs. $62 \%$; $<<0.001)$ or obese ( $41 \%$ vs. $34 \%$; $<0.01$ ). Fewer women than men were regularly physically active ( $35 \%$ vs. $49 \%$; $<<0.001$ ). More women than men had a history of depression ( $17 \%$ vs. $7 \%$; $<<0.0001)$.

During the CR programme, similar number of recurrent events occurred in women and in men (9.6\% vs. 9.0\%). Dropout rates did not differ between women and men (14\% vs. $15 \%$ ). On CR programme completion, women experienced similar improvement of physical exercise capacity in watts ( $22 \pm 18$ vs. $25 \pm 23)$.

\subsection{Documentation and data availability}

Availability of data was limited and varied between the participating countries (Tables 2-4). This is exemplified by the data availability of the item in patients' history "low/no physical activity" (Table 2) ranging from $54 \%$ (Denmark) to $100 \%$ (Croatia, Spain), and the item "exercise capacity - change during CR", ranging from 0\% (Denmark, Hungary, Portugal, Russia, Spain) to 78\% (Switzerland) (Table 4). Moreover, change of smoking behaviour during CR has not routinely been assessed. Therefore no reliable data could be gained from this item too.

\section{Discussion}

To the best of our knowledge, EuroCaReD presents the first eCRF based international registry of baseline patient characteristics, indications, treatment and outcomes in "all-comers" admitted to a CR programme in Europe. Beyond assessment, clinical registry programmes provide an important tool to monitor quality of healthcare services and thereby improve clinical outcome. Through registry evaluation, more effective and efficient systems of CR delivery can be detected. This supports a wider coverage of those patients who can benefit in the future [11]. As a continuation of the previous Carinex and ECRIS project the major tasks of EuroCaReD were:

1. to test the feasibility of a web-based eCRF for collecting clinical baseline and outcome parameters in patients surpassing a CR programme and

2. to define measures that can serve as a benchmark to improve guideline adherence and treatment quality of CR in Europe.

\subsection{Heterogeneity of CV risk factor profiles across European countries}

As EuroCaReD baseline data show, the four most common CV risk factors in patients referred to CR across Europe are hyperlipidaemia, hypertension, low/no physical activity and cigarette smoking. This observation matches with the recently published EuroAspire IV study [15]. As in EuroAspire IV, in EuroCaReD the distribution of the individual risk factors in patients' history considerably varied between the countries. But EuroCaReD findings could not confirm observations of EuroAspire IV that large proportions of CAD patients do not achieve therapeutic targets for $\mathrm{CV}$ disease prevention. In the EuroCaReD population most patients reported adequate prescription of medication even before admission to CR. The different study population could explain this. Whereas in EuroAspire IV patients without documented CAD were asked about their medication, in EuroCaReD all patients presented after a CAD event. Obviously, adequate prescription of cardio-protective medication following evidence-based guidelines is considered more important in secondary than in primary prevention of CAD.

\subsection{Unity and diversity of $C R$ patient baseline clinical parameters in differ- ent European countries}

As in most other reports EuroCaReD overall results show that patients with CAD represented the majority of CR candidates. In accordance with the guidelines for the clinical management of patients with CAD [14], the most common initiating events in patients referred for $\mathrm{CR}$ in European countries are ACS and elective PCI, followed by patients after CABG. But the number of patients admitted for $C R$ because of CHF is surprisingly low and does not meet the requirements given by the current guidelines. Looking to the numbers in various European countries, despite of the current guidelines issued by the European Society of Cardiology [14] the distribution of clinical events initiating CR exhibited a considerable heterogeneity whereas ACS including NSTEMI and STEMI as an important indication for CR was adequately recognised only in Switzerland, Portugal and Germany. In some countries NSTEMI patients were not represented at all (e.g. Greece). Obviously because revascularisation strategies of CAD in Eastern European countries tend more to surgery, in Croatia and Russia patients after CABG represented the largest group of $\mathrm{CR}$ patients. Thus EuroCaReD results show lack of guideline adherence in terms of the most important indication for CR in several European countries.

\subsection{Heterogeneity of $C R$ programme delivery across European countries}

As shown in ECRIS before, a surprisingly wide spread in CR programme content and length has to be noted by EuroCaReD across European countries. Whereas in Eastern Europe and Central Europe, except Austria and Germany where outpatient CR is becoming more common, a short in-patient CR setting has emerged, most other European countries contrast with the tradition of the out-patient setting, which in general is offered over a longer period. The best scientific evidence of improvement in morbidity and mortality in the literature is found for programmes with more sessions and longer duration [16]. Therefore prompted by ECRIS and now by EuroCaReD, across Europe consensus should be found in future on programme content and duration, as well as on patient characteristics for each CR setting. This should be based on scientific evidence or specific medical needs, independent of the development of traditional forms of $\mathrm{CR}$, influenced by local particularities.

\subsection{CR programme patient adherence throughout Europe}

In the EuroCaReD study sites, only about $15 \%$ of patients did not complete the CR programme, mostly because of patient related reasons rather than medical complications. In a questionnaire study from Denmark, authors found 21\% drop-out, with deterioration of physical conditions, lack of time, long distance from residence to hospital, transport problems and lack of understanding of the benefits of CR as the main determinants for drop-out [17]. In another more recently published report from Canada, in accordance to EuroCaReD results the reason for premature termination was most often due to patient dropout (87\%) [18]. These observations provide targets to address compliance problems in CR programmes. In particular, patients who have to interrupt their programme because of medical interventions should be readmitted to CR as soon as possible. As the drop-out risk in EuroCaReD institutions was also high in patients with comorbidities, these subgroups should receive special attention. The high percentage of patients with unspecified reasons of CR programme interruption in EuroCaReD 
needs further qualitative investigation to develop descriptive categories for future study.

\subsection{CR programme success in European countries}

The assumption that a CR programme was successful for the patient has to be weighted in consideration to the targets that the institution has defined with the patient based on his individual needs, CV risk profile and the current guidelines. Because all participation institutions offered exercise based CR, in EuroCaReD CR success was provisionally defined as an increase of exercise capacity of a minimum of $25 \mathrm{~W}$ at the end of programme. By this definition at least more than half of the subgroup of patients with repeat exercise tests during CR successfully completed their programme. Not surprisingly, patients aged $<50$ years, patients being employed and, because of low exercise capacity before programme start, patients after CABG or after STEMI had the highest success rate. Between countries the improvement of exercise capacity varied considerably, obviously because of wide spread in CR programme content and length. But mean improvement of exercise capacity during CR throughout European countries was comparable with results of other reports $[18,19]$.

\subsection{Changes of individual risk factors during CR programme}

Only small improvement in CV risk factors, most likely because of the high pre-medication rate in lipid lowering, blood pressure and diabetes treatment could be documented. In our experience, many patients entered $C R$ very soon after the initiating event and in this situation the medication was still optimally prescribed. Thus patients entering $\mathrm{CR}$ presented lipid levels ranging in the treatment targets and further lowering would not be expected by CR programme activities. So a major task of $C R$ is to get patients to maintain medication and other preventive interventions and treatments by education and motivation. Consistently the EuroAspire III study results show that control of smoking and the use of cardio-protective medication works better in patients who attend a CR programme [20]. EuroCaReD results confirmed these observations. The decrease in number of cigarettes smoked during the CR time period can be rated as a positive effect because there is a linear relationship between number of cigarettes smoked and residual risk [21]. Because of the short observation period EuroCaReD results could not provide a final evaluation on adherence of $C R$ programme effects.

\subsection{Continuation of secondary prevention after finishing CR programme}

To reach long lasting effects of the CR programme on CV risk factors and physical exercise performance effects, long-term care models are crucial. Post CR disease management programmes have been established in many European countries. In three-quarters of the countries that participated in the ECRIS registry, cardiologists are responsible for post-CR patient care often in collaboration with a specialist in internal medicine and/or physiotherapist [12]. The EuroCaReD results reflect this observation. Because of their structured offering it would be advantageous if more than the current level of less than $10 \%$ of post CR patients could be admitted to heart groups supervised by an exercise therapist depending from the country where CR patients are living.

\subsection{Comparison of EuroCaReD patient characteristics with other CR surveys}

In accordance with other registries in- and outside Europe, EuroCaReD results show that much more men than women are admitted to $\mathrm{CR}$ programmes. EuroCaReD findings, demonstrating equal CR benefit for women and men, highlight the need of measures to increase the participation rate of women in CR programmes. In accordance to other parts of the world most patients are admitted for CR because of CAD. But at least in Western Europe more patients after ACS are admitted ( $40 \%$ of all admitted) as e.g. in Canada (20\% of all admitted) [18]. Therefore EuroCaReD patient characteristics and indications for CR correspond more to recently published reports that show the prevalence of ACS as the CR initiating event $[19,22]$. In comparison to the experience of the national investigators of the ECRIS survey, EuroCaReD reported most patients after ACS admitted to CR, but similarly few patients with CHF [12]. Thus EuroCaReD results confirm findings reported in other surveys that CHF patients are underrepresented in exercisebased CR programmes [23]. Although in CHF patients, exercise-based CR does not significantly decrease the risk of all-cause and CV mortality [24], but it reduces hospital readmissions and confers important improvements in health-related quality of life $[25,26]$. Therefore much more CHF patients should be admitted in CR programmes than currently practiced.

\subsection{Strengths and limitations of the study}

In terms of strengths, the EuroCaReD prospective study documents clinical characteristics, treatments and programme outcomes of patients admitted to $\mathrm{CR}$ in different European countries representing usual care across Europe. The registry covers a large population, providing an overview over similarities but also heterogeneity of $\mathrm{CR}$ programmes. Because of the standardised data collection following the CARDS system, the EuroCaReD eCRF can be used as a benchmark throughout European countries. This should enable the CR community to establish more uniform programmes with unified objectives to achieve better treatment results.

This study has several limitations. Availability of data was limited and varied between the participating countries. Therefore caution is warranted when interpreting the data, primarily due to generalizability. In terms of generalizability it has also to be noted that our findings are limited to only 12 European countries where CR institutions were able to contribute data. There is also a possibility that CR centres participating in the survey are most motivated regarding quality assessment and more adherent to evidence-based guidelines. This might result in a selection bias.

Lessons from the limitations of this study caution against lengthy data collection aspirations although the source of collection is a routine clinical programme. EuroCaReD eCRF data collection shows that implementing of electronic reporting alone is not enough relief to achieve sufficient data. To collect high quality data, a robust audit system seems to be crucial. Extra scrutiny should be applied to units where the data collection processes appear to be inadequate.

\section{Conclusions}

To test guideline adherence and treatment quality of CR in Europe this survey provides data of "all-comers" admitted to a CR programme in 12 European countries. Overall patient baseline characteristics at CR programme admission meet the evidence-based guidelines. This also applies to the cardio-protective medication prescription before admission to CR. Nevertheless there are still differences in guideline adherence between countries. Underutilization of CR could be discovered in Europe especially in women and patients with CHF, and in some countries in ACS patients too. This clearly demonstrates a lack of evidencebased guideline adherence. Therefore subgroups where the benefit of CR is underestimated should be specially targeted even because the adherence of patients to CR programmes after admission is high. Deficits could also be found in the uniformity of CR programmes throughout Europe. The heterogeneity of programme design and duration requires better measures that lead to better treatment results, particularly because this first application of eCRF documentation of CR results in European countries was not sufficient enough. 


\section{Funding}

This work was supported by a research grant of the European Association for CV Prevention and Rehabilitation, an association of the European Society of Cardiology, European Heart House, Sophia Antipolis, France.

\section{Conflict of interest}

None declared.

\section{Appendix 1}

EuroCaReD steering committee:

Hannah McGee (chair)

Werner Benzer (EuroCaReD principal investigator)

Birna Bjarnason-Wehrens

Bernhard Rauch

Ann-Dorthe Zwisler

EuroCaReD national coordinators:

Austria: Werner Benzer

Belgium: Paul Dendale

Croatia: Bojan Miletic

Denmark: Ann Dorthe Zwisler

Germany: Bernhard Rauch

Greece: Constantinos H. Davos

Hungary: Attila Simon

Portugal: Ana Abreu

Romania: Dan Gaita

Russia: Nana Pogosova

Spain: Esteban Porrero

Switzerland: Jean-Paul Schmid

Data evaluation and biometry:

Taoufik Ouarrak and Steffen Schneider, Institut für Herzinfarktfoschung, IHF; Ludwigshafen, Bremserstr. 79, D-67063 Ludwigshafen am Rhein, Germany.

EuroCaReD site investigators and cardiac rehabilitation centres

Austria

Axel Philippi, Reha-Sport-Institut, Feldkirch; Karl Mayr, Centrum für Lebensstilmedizin, Linz; Hanns Harpf, Zentrum für ambulante Rehabilitation, Graz; Gerald Zenker, Physikalisches Ambulatorium, Bruck a.d. Mur; Bernhard Eber, Cardio-Vital, Wels.

Belgium

Jan Berger, Jessa Ziekenhuis, Hasselt; Benedicte Heyndrickx, University Hospital, Brussels; Catherine Demaeyer, University Hospital, Antwerpen; Cathy Kuppens, Mariaziekenhuis, Noord-Limburg, Overpelt; Christophe Laruell, Clinique St Luc, Bouge; Hans Vandekerckhove, University Hospital, Gent; Hubert Dereppe, Centre Hospitalier de Wallonie picarde, Tournai; Ivan Elegeert, AZ Ziekenhuis, Groeninge; Jamon Alexander, Erasme Hospital, Brussels; Joelle Deconinck, Cliniques de l'Europe, site Saint-Michel, St-Michel à Etterbeek; Johan Desutter, AZ Maria, Middelares; Kathleen Retailleau, C.H.U, Charleroi; Koen Kerremans, Imelda Ziekenhuis, Bonheiden; Laurence Gabriel, Universite catholique, Leuven; Michel Deceuninck, Heilig Hartziekenhuis, Roeselare; Michel Lamotte, Universite Libre, Brussels; Patrick Willems, AZ Onze-Lieve-Vrouw Ter Linden; Paul Beckers, Universitair Ziekenhuis, Antwerpen; Tommy Mulleners, St. Fransiscusziekenhuis, Heusden; Wilfried Mullens, Ziekenhuis Oost-Limburg, Genk.

Croatia

Bojan Miletic, Clinic for Rehabilitation and Prevention of CV Diseases, Opatija.

Denmark

Ann-Dorthe Zwisler, Rigshospitalet, Copenhagen; Marianne Frederiksen, Bispebjerg Hospital, Copenhagen; Irene Wernberg, Gentofte Hospital, Hellerup; Jens Rokkedal, Glostrup Hospital, Glostrup; Bettina Rodriguez, NorthZealands Hospital, Hillerød; Ulla Overgaard
Andersen, Holbaek Hospital, Holbaek; Charlotte Helmark, Roskilde Hospital, Roskilde; Britta Nielsen, Nykoebing Falster Hospital, Nykoebing Falster; Kristian Korsgaard Thomsen, Hospital of Southwest Jutland, Esbjerg; Monica Petronela Poenaru, Lillebaelt Hospital Vejle; Connie Bonde Moeller, Odense University Hospital Odense; Vibeke Hougaard, Regional Hospital, Randers; Dorrit Grosen Andersen, Aarhus University Hospital, Aarhus; Susanne Riis Christiansen, Aalborg University Hospital, Aalborg; Ole May, Regional Hospital Herning.

Germany

Gerhard Boenner, Park Klinikum und Klinik Lazariterhof, Bad Krozingen; Albrecht Charrier, Alice Park Reha, Darmstadt; Roland Fries, Gotthard-Schettler-Klinik, Bad Schönborn; Manju Guha, Reha-Klinik am Sendesaal, Breme; Eike Hoberg, Mühlenbergklinik Holsteinische Schweiz, Bad Malente-Gremsmühlen; Marthin Karoff, Klinik Königsfeld, Ennepetal; Bernhard Rauch, ZAR - Ludwigshafen Klinikum, Ludwigshafen; Wolfgang Mayer-Berger, Klinik Roderbirken, Leichlingen; Klaus Schröder, Zentrum für ambulante Rehabilitation, Stuttgart; Rainer Schubmann, Dr. Becker Klinik Möhnesee, Möhnesee; Heinz Voeller, Klinik am See, Rüdersdorf b. Berlin.

Greece

Evangelia Kouidi, Aristotle University of Thessaloniki, Thessaloniki; Apostolos Karavidas, Athens General Hospital, Athens; Christina Chrysohoou, Hippokratio University Hospital, Athens; Yannis Laoutaris, Onassis Cardiac Surgery Centre, Kallithea.

Hungary

Attila Simon State Hospital for Cardiology, Balatonfured; Eva Simon,

Cardiac Rehabilitation Department, Sopron Rehabilitation Centre.

Portugal

Afonso Rocha, Hospital S. João, Porto, Sofia Viamonte, Hospital S. António, Porto; Miguel Mendes, Hospital de Santa Cruz, Lisbon; Ana Abreu, Hospital Santa Marta, Lisbon.

Romania

Dan Gaita, Cardiac Rehabilitation Clinic, Timisoara.

Russia

Nana Pogosova, Olga Sokolova, Yulia Yufereva, National Research Centre for Preventive Medicine, Moscow; Irina Osipova, Altai State Medical University, Barnaul; Irina Ryamzina, Perm State Medical Academy, Perm.

Spain

Esteban García Porrero, Cardiologia No Invasiva, Fisiocard, Leon.

Switzerland

Jean-Paul Schmid, Kardiale Rehabilitation, Inselspital, Bern; Beat Schaer, Kardiale Rehabilitation, Winterthur; Lorenz Felder, Ambulante Herzrehabilitation, Zürich; Stephanie Kiencke, Universitätsspital, Basel; Gudrun Haager, Kantonspital, St. Gallen.

\section{References}

[1] European Heart Network and European Society of CardiologyEur. Cardiovasc. Dis. Stat. (2012)

[2] N. Townsend, et al., Cardiovascular disease in Europe - epidemiological update 2015, Eur. Heart J. 36 (40) (2015) 2696-2705.

[3] E.S. Ford, et al., Explaining the decrease in U.S. deaths from coronary disease, 1980 2000, N. Engl. J. Med. 356 (23) (2007) 2388-2398.

[4] H.M. Krumholz, et al., Report of the National Heart, Lung, and Blood Institute working group on outcomes research in cardiovascular disease, Circulation 111 (23) (2005) 3158-3166.

[5] G.J. Balady, et al., Referral, enrollment, and delivery of cardiac rehabilitation/secondary prevention programs at clinical centers and beyond: a presidential advisory from the American Heart Association, Circulation 124 (25) (2011) 2951-2960.

[6] L. Anderson, et al., Exercise-based cardiac rehabilitation for coronary heart disease: cochrane systematic review and meta-analysis, J. Am. Coll. Cardiol. 67 (1) (2016) 1-12.

[7] E. Davies, et al., Exercise based rehabilitation for heart failure, Cochrane Database Syst. Rev. (2010).

[8] B.S. Heran, et al., Exercise-based cardiac rehabilitation for coronary heart disease, Cochrane Database Syst. Rev. (7) (2011), Cd001800.

[9] F.G. Kushner, H. M., S. SC Jr., et al., ACC/AHA guidelines for the management of patients with ST-elevation myocardial infarction (updating the 2004 guideline and 2007 focused update) and ACC/AHA/SCAI guidelines on percutaneous coronary 
intervention (updating the 2005 guideline and 2007 focused update) a report of the American College of Cardiology Foundation/American Heart Association Task Force on Practice Guidelines, J. Am. Coll. Cardiol. 54 (2009) 2205-2241.

[10] P.G. Steg, J. S., et al., ESC Guidelines for the management of acute myocardial infarction in patients presenting with ST-segment elevation, Eur. Heart J. 33 (2012) 2569-2619.

[11] L. Vanhees, et al., A representative study of cardiac rehabilitation activities in European Union Member States, J. Cardpulm. Rehabil. 22 (2002) 264-272.

[12] B. Bjarnason-Wehrens, et al., Cardiac rehabilitation in Europe: results from the European Cardiac Rehabilitation Inventory Survey, Eur. J. Cardiovasc. Prev. Rehabil. 17 (4) (2010) 410-418.

[13] M.R. Flynn, et al., European data standards for clinical cardiology practice: the Cardiology Audit and Registration Data Standards (CARDS), Eur. Heart J. 26 (3) (2005) 308-313.

[14] M.F. Piepoli, U. Corra, S. Adamopoulos, Secondary prevention in the clinical management of patients with cardiovascular diseases. Core components, standards and outcome measures for referral and delivery: a policy statement from the cardiac rehabilitation section of the European Association for Cardiovascular Prevention \& Rehabilitation. Endorsed by the Committee for Practice Guidelines of the European Society of Cardiology, Eur. J. Prev. Cardiol. 21 (6) (2014) 664-681.

[15] K. Kotseva, D. Wood, G. De Backer, EUROASPIRE IV: a European Society of Cardiology survey on the lifestyle, risk factor and therapeutic management of coronary patients from 24 European countries, Eur. J. Prev. Cardiol. 23 (6) (2016 Apr) 636-648.

[16] J.A. Doll, et al., Effectiveness of cardiac rehabilitation among older patients after acute myocardial infarction, Am. Heart J. 170 (5) (2015) 855-864.
[17] T. Mikkelsen, K. Korsgaard Thomsen, O. Tchijevitch, Non-attendance and drop-out in cardiac rehabilitation among patients with ischaemic heart disease, Dan. Med. J. 61 (10) (2014) A4919.

[18] S.L. Grace, et al., The Canadian Cardiac Rehabilitation Registry: inaugural report on the status of cardiac rehabilitation in Canada, Rehabil Res Pract 2015 (2015) 278979.

[19] J. Niebauer, et al., Long-term effects of outpatient cardiac rehabilitation in Austria: a nationwide registry, Wien. Klin. Wochenschr. 126 (5-6) (2014) 148-155.

[20] K. Kotseva, et al., Use and effects of cardiac rehabilitation in patients with coronary heart disease: results from the EUROASPIRE III survey, Eur. J. Prev. Cardiol. 20 (5) (2013) 817-826.

[21] J.A. Kim, et al., Relationship between amount of cigarette smoking and coronary atherosclerosis on coronary CTA in asymptomatic individuals, Int. J. Card. Imaging 29 (Suppl. 1) (2013) 21-28.

[22] R. Nebel, et al., Age-dependency of clinical characteristics of patients participating cardiovascular rehabilitation: results from the German Registry of Ambulatory Cardiac Rehabilitation - KARREE, Open J. Thearpy and Rehabil. 2 (2014) 207-216.

[23] M.F. Piepoli, et al., ExtraHF survey: the first European survey on implementation of exercise training in heart failure patients, Eur. J. Heart Fail 17 (6) (2015) 631-638.

[24] C.M. O'Connor, et al., Efficacy and safety of exercise training in patients with chronic heart failure: HF-ACTION randomized controlled trial, JAMA 301 (14) (2009) 1439-1450.

[25] V.A. Sagar, et al., Exercise-based rehabilitation for heart failure: systematic review and meta-analysis, Open Heart 2 (1) (2015), e000163.

[26] R.S. Taylor, et al., Exercise-based rehabilitation for heart failure, Cochrane Database Syst. Rev. 4 (2014) Cd003331. 